Research Article

\title{
Composition Operators on Some Banach Spaces of Harmonic Mappings
}

\author{
Munirah Aljuaid ${ }^{1}$ and Flavia Colonna $\mathbb{D}^{2}$ \\ ${ }^{1}$ Department of Mathematics, Northern Borders University, Arar 73222, Saudi Arabia \\ ${ }^{2}$ Department of Mathematical Sciences, George Mason University, Fairfax, VA 22030, USA
}

Correspondence should be addressed to Flavia Colonna; fcolonna@gmu.edu

Received 26 November 2019; Accepted 17 December 2019; Published 19 February 2020

Academic Editor: Stanislav Hencl

Copyright $\odot 2020$ Munirah Aljuaid and Flavia Colonna. This is an open access article distributed under the Creative Commons Attribution License, which permits unrestricted use, distribution, and reproduction in any medium, provided the original work is properly cited.

We study the composition operators on Banach spaces of harmonic mappings that extend several well-known Banach spaces of analytic functions on the open unit disk in the complex plane, including the $\alpha$-Bloch spaces, the growth spaces, the Zygmund space, the analytic Besov spaces, and the space BMOA.

\section{Introduction}

Given a simply connected region $\Omega$ in the complex plane $\mathbb{C}$, a harmonic mapping with domain $\Omega$ is a complex-valued function $h$ defined on $\Omega$ satisfying the Laplace equation:

$$
\Delta h:=4 h_{z \bar{z}} \equiv 0, \quad \text { on } \Omega \text {, }
$$

where $h_{z \bar{z}}$ is the mixed complex second partial derivative of $h$.

It is well known that a harmonic mapping $h$ admits a representation of the form $f+\bar{g}$, where $f$ and $g$ are analytic functions. This representation is unique if, for a fixed a base point $z_{0}$ in the domain, the function $g$ is chosen so that $g\left(z_{0}\right)=0$.

In this paper, we shall assume all the functions under consideration are defined on $\mathbb{D}=\{z \in \mathbb{C}:|z|<1\}$. Let us denote $\mathscr{H}(\mathbb{D})$ as the set of harmonic mappings on $\mathbb{D}, H(\mathbb{D})$ as the set of analytic functions on $\mathbb{D}, S(\mathbb{D})$ as the set of analytic self-maps of $\mathbb{D}$, and $A u t(\mathbb{D})$ as the group of (conformal) disk automorphisms of $\mathbb{D}$.

Given an analytic self-map $\varphi$ of $\mathbb{D}$, the composition operator induced by $\varphi$ is defined as the operator

$$
C_{\varphi} f=f \circ \varphi
$$

for all $f$ belonging to a selected class. It is immediate to see that such an operator preserves harmonic mappings.

Since analytic functions are clearly harmonic, an interesting question is how to extend to harmonic mappings
Banach space structures of known spaces of analytic functions $X$ in such a way that the norm on the larger space agrees with the norm of $X$ when restricting to the elements of $X$.

An example of a space of harmonic mappings on $\mathbb{D}$ that extends a Banach space of analytic functions is $B M O H$, defined as the space of harmonic mappings on $\mathbb{D}$ which are Poisson integrals of functions on the unit circle $\partial \mathbb{D}$ belonging to BMO, which was thoroughly studied by Girela [1]. In that work, it was shown that $H(\mathbb{D}) \cap \mathrm{BMOH}$ is the space BMOA of analytic functions of bounded mean oscillation.

In [2], the first author pursued this study by extending several classes of Banach spaces, including the Bloch space $\mathscr{B}$ and its generalizations $\mathscr{B}^{\alpha}$ known as $\alpha$-Bloch spaces introduced by Zhu in [3], the growth spaces $\mathscr{A}^{-\alpha}$ (where $\alpha>0$ ), the Zygmund space $\mathscr{Z}$, and the analytic Besov spaces $B^{p}$ for $p>1$. In particular, the linear structure and properties of the harmonic $\alpha$-Bloch spaces $\mathscr{B}_{H}^{\alpha}$, the harmonic growth spaces $\mathscr{A}_{H}^{-\alpha}$ (for $\alpha>0$ ), and the harmonic Zygmund space $\mathscr{Z}_{H}$ were studied in $[4,5]$. The harmonic Besov spaces $B_{H}^{p}$ for $p>1$ were introduced in [2].

In this work, after giving in Section 2 some preliminaries on the spaces of harmonic mappings mentioned above, we introduce the harmonic Besov space $B_{H}^{1}$ and an alternative extension of BMOA to harmonic mappings denoted by $\mathrm{BMOA}_{H}$. We then analyze the composition operators acting on all such spaces. Specifically, we characterize the composition operators that are bounded, compact, or bounded 
below and identify the isometries for most of them. For each of these spaces, we also examine the eigenfunctions of the composition operators.

Let us denote $X_{H}$ as a harmonic extension of the Banach space $X$ of analytic functions whose corresponding norms coincide on the elements of $X$. For the spaces treated in this work, due to simple estimates connecting the seminorm of a harmonic mapping in $X_{H}$ to the seminorm of the associated analytic and antianalytic (i.e., conjugate of analytic) components in $X$, it turns out that the composition operator $C_{\varphi}$ acting on $X_{H}$ is bounded (respectively, compact, bounded below, closed range) if and only if $C_{\varphi}$ acting on $X$ is bounded (respectively, compact, bounded below, closed range). This result will be a consequence of a general theorem proved in Section 3 .

In Section 4, we focus on the study of the isometries among the composition operators. Finally, in Section 5, we study the eigenfunctions of $C_{\varphi}$ acting on the harmonic spaces $\mathscr{B}_{H}^{\alpha}, \mathscr{A}_{H}^{-\alpha}, \mathscr{Z}_{H}, B_{H}^{p}$, and $\mathrm{BMOA}_{H}$.

\section{Harmonic Spaces Treated in This Work}

2.1. Harmonic $\alpha$-Bloch Spaces. For $\alpha>0$, the harmonic $\alpha--$ Bloch space $\mathscr{B}_{H}^{\alpha}$ is the collection of all $h \in \mathscr{H}(\mathbb{D})$ such that

$$
\beta_{h}^{\alpha}:=\sup _{z \in \mathbb{D}}\left(1-|z|^{2}\right)^{\alpha}\left(\left|h_{z}(z)\right|+\left|h_{\bar{z}}(z)\right|\right)<\infty .
$$

The mapping $h \longmapsto\|h\|_{\mathscr{B}_{H}^{\alpha}}:=|h(0)|+\beta_{h}^{\alpha}$ defines a Banach space structure on $\mathscr{B}_{H}^{\alpha}$. This space is an extension to harmonic mappings of the (analytic) $\alpha$-Bloch space $\mathscr{B}^{\alpha}$ introduced by Zhu in [3]. We recall that an analytic function $f$ belongs to $\mathscr{B}^{\alpha}$ if and only if

$$
\beta_{f}^{\alpha}:=\sup _{z \in \mathbb{D}}\left(1-|z|^{2}\right)^{\alpha}\left|f^{\prime}(z)\right|<\infty,
$$

with norm $\|f\|_{\mathscr{B}^{\alpha}}=|f(0)|+\beta_{f}^{\alpha}$. Thus, representing $h \in \mathscr{H}(\mathbb{D})$ as $f+\bar{g}$ with $f, g \in H(\mathbb{D})$ and $g(0)=0$, we see that $h_{z}=f^{\prime}$ and $h_{\bar{z}}=\overline{g^{\prime}}$. Hence, $\beta_{h}^{\alpha}=\sup _{z \in \mathbb{D}}\left(1-|z|^{2}\right)^{\alpha}\left(\left|f^{\prime}(z)\right|+\left|g^{\prime}(z)\right|\right)$, and

$$
\frac{1}{2}\left(\beta_{f}^{\alpha}+\beta_{g}^{\alpha}\right) \leq \max \left\{\beta_{f}^{\alpha}, \beta_{g}^{\alpha}\right\} \leq \beta_{h}^{\alpha} \leq \beta_{f}^{\alpha}+\beta_{g}^{\alpha}
$$

Consequently, a harmonic mapping $h$ belongs to $\mathscr{B}_{H}^{\alpha}$ if and only if the unique functions $f$ and $g$ analytic on $\mathbb{D}$ such that $h=f+\bar{g}$ with $g(0)=0$ are in $\mathscr{B}^{\alpha}$ (for more information on the spaces $\mathscr{B}_{H}^{\alpha}$, see [4]).

For $\alpha=1$, the space $\mathscr{B}^{\alpha}$ is the classical Bloch space $\mathscr{B}$, and the corresponding harmonic extension will be denoted by $\mathscr{B}_{H}$. The elements of this space were first studied in [6].

2.2. Harmonic Growth Spaces. For $\alpha>0$, the harmonic growth space $\mathscr{A}_{H}^{-\alpha}$ is the collection of all harmonic mappings $h$ on $\mathbb{D}$ such that

$$
\|h\|_{\mathscr{A}_{H}^{-\alpha}}:=\sup _{z \in \mathbb{D}}\left(1-|z|^{2}\right)^{\alpha}|h(z)|<\infty .
$$

The mapping $h \longmapsto h_{\mathscr{A}_{H}^{-\alpha}}$ defines a Banach space structure on $\mathscr{A}_{H}^{-\alpha}$, which again extends that of the (analytic) growth space $\mathscr{A}^{-\alpha}$. For $\alpha>0$, the functions $f, g \in \mathscr{A}^{-\alpha}$ if and only if $f+\bar{g} \in \mathscr{A}_{H}^{-\alpha}$ (see $[2,4]$ for details; for more information on the analytic growth space, see [7]).

2.3. Harmonic Zygmund Space. Recall that the Zygmund space is the space $\mathscr{Z}$ consisting of the analytic functions $f$ on $\mathbb{D}$ such that $f^{\prime} \in \mathscr{B}$ with norm

$$
\|f\|_{\mathscr{Z}}:=|f(0)|+\left|f^{\prime}(0)\right|+\sup _{z \in \mathbb{D}}\left(1-|z|^{2}\right)\left|f^{\prime \prime}(z)\right| .
$$

The harmonic Zygmund space, introduced in [2], is the collection of harmonic mappings $h$ on $\mathbb{D}$ such that $h_{z}+h_{\bar{z}} \in \mathscr{B}_{H}$. Define

$$
\begin{aligned}
\|h\|_{\mathscr{X}_{H}}:= & |h(0)|+\left|h_{z}(0)\right|+\left|h_{\bar{z}}(0)\right|+\beta_{h_{z}+h_{\bar{z}}} \\
= & |h(0)|+\left|h_{z}(0)\right|+\left|h_{\bar{z}}(0)\right|+\sup _{z \in \mathbb{D}}\left(1-|z|^{2}\right) \\
& \cdot\left(\left|h_{z z}(z)\right|+\left|h_{\overline{z z}}(z)\right|\right) .
\end{aligned}
$$

The mapping $h \longmapsto\|h\|_{\mathscr{E}_{H}}$ defines a Banach space structure on $\mathscr{Z}_{H}$. The harmonic mapping $h$ belongs to $\mathscr{Z}_{H}$ if and only if the unique functions $f$ and $g$ analytic on $\mathbb{D}$ such that $h=f+\bar{g}$ with $g(0)=0$ are in $\mathscr{Z}$. Furthermore,

$$
\frac{1}{2}\left(f_{\mathscr{Z}}+g_{\mathscr{E}}\right) \leq\|h\|_{\mathscr{E}_{H}} \leq\|f\|_{\mathscr{Z}}+g\|g\|_{\mathscr{Z}} \text {. }
$$

In [5], it is shown that the elements of the space $\mathscr{Z}_{H}$ can be characterized in terms of the membership to the classical Zygmund class, and the corresponding norms are equivalent.

2.4. Harmonic Besov Spaces. For $1<p<\infty$, a harmonic mapping $h$ in $\mathbb{D}$ is said to belong to the harmonic Besov space $B_{H}^{p}$ if the quantity

$$
b_{p}(h):=\left(\int_{\mathbb{D}}\left(1-|z|^{2}\right)^{p-2}\left(\left|h_{z}(z)\right|+\left|h_{\bar{z}}(z)\right|\right)^{p} \mathrm{~d} A(z)\right)^{1 / p},
$$

is finite, where $\mathrm{d} A(z)$ is the normalized area measure on $\mathbb{D}$ such that $A(\mathbb{D})=1$.

The mapping $h \longmapsto b_{p}(h)$ defines a Möbius-invariant seminorm, and $h \longmapsto\|h\|_{B_{H}^{p}}:=|h(0)|+b_{p}(h)$ defines a Banach space structure on $B_{H}^{p}$ which extends the ordinary norm on $B^{p}$. As shown in [2], for $1<p<\infty$, two analytic functions $f$ and $g$ on $\mathbb{D}$ belong to $B^{p}$ if and only if $f+\bar{g} \in B_{H}^{p}$. Moreover, if $g(0)=0$, then

$\frac{1}{2}\left(\|f\|_{B^{p}}+\|g\|_{B^{p}}\right) \leq\|f+\bar{g}\|_{B_{H}^{p}} \leq 2\left(\|f\|_{B^{p}}+\|g\|_{B^{p}}\right)$.

Recall that in the analytic case, the Besov space $B^{2}$ is the Dirichlet space $\mathscr{D}$, which is a Hilbert space under an equivalent norm, namely,

$$
\|f\|_{\mathscr{D}}:=\left(|f(0)|^{2}+\int_{\mathbb{D}}\left|f^{\prime}(z)\right|^{2} \mathrm{~d} A(z)\right)^{1 / 2} .
$$

Likewise, the harmonic Besov space for $p=2$, which we call the harmonic Dirichlet space and denote by $\mathscr{D}_{H}$, can be endowed with a Hilbert space structure via the inner product: 


$$
\begin{aligned}
\left\langle h_{1}, h_{2}\right\rangle_{\mathscr{D}_{H}}:= & h_{1}(0) \overline{h_{2}(0)}+\int_{\mathbb{D}}\left(\left(h_{1}\right)_{z}(z) \overline{\left(h_{2}\right)_{z}(z)}\right. \\
& \left.+\left(h_{1}\right)_{\bar{z}}(z) \overline{\left(h_{2}\right)_{\bar{z}}(z)}\right) \mathrm{d} A(z),
\end{aligned}
$$

whose associated norm

$$
h_{\mathscr{D}_{H}}=\left(|h(0)|^{2}+\int_{\mathbb{D}}\left(\left|h_{z}(z)\right|^{2}+\left|h_{\bar{z}}(z)\right|^{2}\right) \mathrm{d} A(z)\right)^{1 / 2} \text {, }
$$

is equivalent to $h_{B_{H}^{2}}$. In particular, if $h \in \mathscr{D}_{H}, h=f+\bar{g}$ with $g(0)=0$, then

$$
\|h\|_{\mathscr{D}_{H}}^{2}=\|f\|_{\mathscr{D}}^{2}+\|g\|_{\mathscr{D}}^{2} .
$$

The analytic Besov space $B^{1}$ is defined as the set consisting of the functions $f \in H(\mathbb{D})$ of the form

$$
f(z)=\sum_{n=1}^{\infty} a_{n} L_{w_{n}}
$$

where $\left(a_{n}\right)$ is absolutely summable, and $w_{n} \in \mathbb{D}$ for $n \in \mathbb{N}$ and for $w \in \mathbb{D}, L_{w}$ is the disk automorphism defined by

$$
L_{w}(z)=\frac{w-z}{1-\bar{w} z}
$$

The norm of $f$ in $B^{1}$ is defined as

$$
f_{B^{1}}:=\inf \sum_{n=1}^{\infty}\left|a_{n}\right|,
$$

where the infimum is taken over all above representations of $f$. Under this norm, $B^{1}$ is a Möbius-invariant Banach space contained in the disk algebra $H^{\infty} \cap C(\overline{\mathbb{D}})$ (see [8] for details).

In [9], it was shown that $B^{1}$ is the smallest Möbius-invariant space, which is why it is commonly known as the minimal Möbius-invariant space. Moreover, $f \in B^{1}$ if and only if

$$
\int_{\mathbb{D}}\left|f^{\prime \prime}(z)\right| \mathrm{d} A(z)<\infty
$$

A more convenient non-Möbius-invariant norm on $B^{1}$ equivalent to $\|\cdot\|_{B^{1}}$ is given by

$$
\|f\|:=\max \left\{|f(0)|,\left|f^{\prime}(0)\right|, \int_{\mathbb{D}}\left|f^{\prime \prime}(z)\right| \mathrm{d} A(z)\right\} .
$$

We introduce the harmonic Besov space $B_{H}^{1}$ as the collection of harmonic mappings $h$ on $\mathbb{D}$ for which

$$
b_{1}(h):=\int_{\mathbb{D}}\left(\left|h_{z z}(z)\right|+\left|h_{\overline{z z}}(z)\right|\right) \mathrm{d} A(z)<\infty .
$$

Representing a harmonic mapping $h$ as $f+\bar{g}$ with $f, g \in \underline{H}(\mathbb{D})$ and $g(0)=0$, we see that $h_{z z}=f^{\prime \prime}$ and $h_{\bar{z} \bar{z}}=\bar{g}^{\prime \prime}$. Thus, $h \in B_{H}^{1}$ if and only if $f, g \in B^{1}$ and

$$
\frac{1}{2}(\|f\|+\|g\|) \leq \max \{\|f\|,\|g\|\} \leq\|h\| \leq\|f\|+\|g\|,
$$

where $\|h\|:=\max \left\{|h(0)|,\left|h_{z}(0)\right|+\left|h_{\bar{z}}(0)\right|+b_{1}(h)\right\}$. Consequently, the set $B_{H}^{1}$ is contained in $\mathscr{H}(\mathbb{D}) \cap C(\overline{\mathbb{D}})$, and a harmonic mapping $h$ belongs to $B_{H}^{1}$ if and only if it admits a representation of the form

$$
h(z)=\sum_{n=1}^{\infty} a_{n} L_{w_{n}}+\sum_{n=1}^{\infty} b_{n} \overline{L_{\zeta_{n}}}
$$

where $\left(a_{n}\right),\left(b_{n}\right) \in \ell_{1}$, and $w_{n}, \zeta_{n} \in \mathbb{D}$ for each $n \in \mathbb{N}$. This leads to the following equivalent Möbius-invariant norm on $B_{H}^{1}$ that extends (18):

$$
\|h\|_{B_{H}^{1}}:=\inf \left\{\sum_{n=1}^{\infty}\left|a_{n}\right|+\sum_{n=1}^{\infty}\left|b_{n}\right|\right\},
$$

where the infimum is taken over all above representations of $h$. Under both norms, $B_{H}^{1}$ is a Banach space and for $h=$ $f+\bar{g}$ with $f, g \in H(\mathbb{D})$

$$
\frac{1}{2}\left(\|f\|_{B^{1}}+\|g\|_{B^{1}}\right) \leq\|h\|_{B_{H}^{1}} \leq\|f\|_{B^{1}}+\|g\|_{B^{1}} .
$$

2.5. Harmonic Space of Bounded Mean Oscillation. Recall that the space BMOA of analytic functions of bounded mean oscillation is the Banach space with norm

$$
\|f\|_{\mathrm{BMOA}}:=|f(0)|+\sup _{a \in \mathbb{D}}\left\|f \circ L_{a}-f(a)\right\|_{H^{2}}<\infty,
$$

and the associated seminorm is Möbius invariant. We recall that the norm of a function $f$ in the Hardy Hilbert space $H^{2}$ is defined as

$$
\|f\|_{H^{2}}=\sup _{0<r<1}\left(\frac{1}{2 \pi} \int_{0}^{2 \pi}\left|f\left(r e^{i \theta}\right)\right|^{2} \mathrm{~d} \theta\right)^{1 / 2} .
$$

It is well known that a function $f \in H(\mathbb{D})$ is in BMOA if and only if

$$
\sup _{a \in \mathbb{D}} \int_{\mathbb{D}}\left|f^{\prime}(z)\right|^{2}\left(1-\left|L_{a}(z)\right|^{2}\right) \mathrm{d} A(z)<\infty,
$$

and $\|\cdot\|_{\mathrm{BMOA}}$ is equivalent to $\|\cdot\|_{*}$ defined by

$$
\|f\|_{*}:=|f(0)|+\sup _{a \in \mathbb{D}}\left(\int_{\mathbb{D}}\left|f^{\prime}(z)\right|^{2}\left(1-\left|L_{a}(z)\right|^{2}\right) \mathrm{d} A(z)\right)^{1 / 2} .
$$

see [1] for a comprehensive analysis of the functions of bounded mean oscillation.

The space $\mathrm{BMOH}$ of harmonic functions of bounded mean oscillation is the collection of harmonic mappings $h$ on $\mathbb{D}$ of the form $h=P[f]$, where $f \in \operatorname{BMO}(\partial \mathbb{D})$ and $P[f]$ is the Poisson integral of $f$. As shown in [1], a norm on this space whose associated seminorm yields the Möbius-invariant seminorm in BMOA when restricted to the analytic functions on $\mathbb{D}$ is given by

$$
\|h\|_{\mathrm{BMOH}}=|h(0)|+\sup _{a \in \mathbb{D}}\left\|h \circ L_{a}-h(a)\right\|_{h^{2}},
$$

where for $h \in \mathscr{H}(\mathbb{D})$,

$$
\|h\|_{h^{2}}=\sup _{0<r<1}\left(\frac{1}{2 \pi} \int_{0}^{2 \pi}\left|h\left(r e^{i \theta}\right)\right|^{2} \mathrm{~d} \theta\right)^{1 / 2} .
$$


If $f$ is analytic, then $\|f\|_{h^{2}}=\|f\|_{H^{2}}$. Representing $h$ as $f+$ $\bar{g}$ with $f, g$ analytic and $g(0)=0$, we see that

$\left\|h \circ L_{a}-h(a)\right\|_{h^{2}} \leq \sqrt{2}\left(\left\|f \circ L_{a}-f(a)\right\|_{H^{2}}+\left\|g \circ L_{a}-g(a)\right\|_{H^{2}}\right)$.
Thus, if $f, g \in \mathrm{BMOA}$, then $f+\bar{g} \in \mathrm{BMOH}$. However, this norm does not lead to a lower bound in terms of the norms of $f$ and $g$. Thus, for $h \in \mathscr{H}(\mathbb{D})$, we define the harmonic extension of the space BMOA as follows:

$$
\|h\|_{H, *}:=|h(0)|+\sup _{a \in \mathbb{D}}\left(\int_{\mathbb{D}}\left(\left|h_{z}(z)\right|^{2}+\left|h_{\bar{z}}(z)\right|^{2}\right)\left(1-\left|L_{a}(z)\right|^{2}\right) \mathrm{d} A(z)\right)^{1 / 2},
$$

if this expression is finite. We see that taking $h=f+\bar{g}$ with $g(0)=0$, so that $h_{z}=f^{\prime}$ and $h_{\bar{z}}=\overline{g^{\prime}}$, we have

$$
\frac{1}{2}\left(\|f\|_{*}+\|g\|_{*}\right) \leq\|f+\bar{g}\|_{H, *} \leq\|f\|_{*}+\|g\|_{*} .
$$

In particular, consistent with the notation we have been using throughout, denoting by $\mathrm{BMOA}_{H}$ the collection of such harmonic mappings, we see that under $\|\cdot\|_{H, *}$, $\mathrm{BMOA}_{H}$ is a Banach space that extends the norm $\|\cdot\|_{*}$ on $\mathrm{BMOA}$. Moreover, $h \in \mathrm{BMOA}_{H}$ if and only if the associated analytic functions $f$ and $g$ belong to BMOA.

It is well known that for $1<p \leq q<\infty, B^{1} \subseteq B^{p} \subseteq$ $B^{q} \subseteq B M O A \subseteq \mathscr{B}$ (e.g., see [10]), and all inclusions are proper. Due to the connection between the respective norms, we see that the same inclusion relations hold for the corresponding harmonic spaces.

\section{General Theorem on Composition Operators}

Let $X_{H}$ be a Banach space of harmonic mappings on $\mathbb{D}$ with seminorm $\|\cdot\|_{s X_{H}}$ and norm

$$
\|h\|_{X_{H}}=|h(0)|+\|h\|_{s X_{H}},
$$

such that the point-evaluation functionals are bounded. Let $X=H(\mathbb{D}) \cap X_{H}$ and denote by $\|\cdot\|_{s X}$ and $\|\cdot\|_{X}$ the seminorm and norm induced on $X$, that is, for $f \in X$, let $\|f\|_{X}=|f(0)|+\|f\|_{s X}=|f(0)|+\|f\|_{s X_{H}}=\|f\|_{X_{H}}$. Assume further that for each $h=f+\bar{g} \in X_{H}$ with $g(0)=0$, the associated analytic functions $f$ and $g$ belong to $X$ and

$$
\|f+\bar{g}\|_{s X_{H}}=\|f\|_{s X}+\|g\|_{s X},
$$

where by $A \asymp B$ we mean $c_{1} A \leq B \leq c_{2} A$ for some positive constants $c_{1}$ and $c_{2}$.

Theorem 1. If $\varphi \in S(\mathbb{D})$, then

(a) $C_{\varphi}: X_{H} \longrightarrow X_{H}$ is bounded if and only if $C_{\varphi}: X \longrightarrow X$ is bounded

(b) $C_{\varphi}: X_{H} \longrightarrow X_{H}$ is compact if and only if $C_{\varphi}: X \longrightarrow X$ is compact

(c) $C_{\varphi}: X_{H} \longrightarrow X_{H}$ is bounded below if and only if $C_{\varphi}: X \longrightarrow X$ is bounded below

(d) $C_{\varphi}: X_{H} \longrightarrow X_{H}$ is closed range if and only if $C_{\varphi}: X \longrightarrow X$ is closed range
Proof. Note that (c) and (d) are equivalent since composition operators are injective. For statements (a), (b), and (c), the implication $\Longrightarrow$ is obvious. To prove that the converse statements hold, assume $C_{\varphi}: X \longrightarrow X$ is bounded. Let $h \in X_{H}$ and let $f$ and $g$ be the associated analytic functions so that $h=f+\bar{g}$ and $g(0)=0$. Then, $f, g \in X$, and by our assumption that $C_{\varphi}$ is bounded on $X$, it follows that $f \circ \varphi, g \circ \varphi \in X$. Thus, $h \circ \varphi \in X_{H}$. By the Closed Graph Theorem, the operator $C_{\varphi}$ is bounded on $X_{H}$.

Next, assume $C_{\varphi}: X \longrightarrow X$ is compact. Let $\left(h_{n}\right)$ be a sequence in $X_{H}$ such that $\left\|h_{n}\right\|_{X_{H}} \leq 1$ and for each $n \in \mathbb{N}$, let $f_{n}, g_{n}$ be the associated analytic functions so that $h_{n}=f_{n}+$ $\overline{g_{n}}$ and $g_{n}(0)=0$. By (36), $\left\|f_{n}\right\|_{s X}+\left\|g_{n}\right\|_{s X} \leq C\left\|h_{n}\right\|_{X_{H}} \leq C$. Moreover, $f_{n}(0)=h_{n}(0)$. Thus, the sequences $\left(f_{n}\right)$ and $\left(g_{n}\right)$ have bounded norms in $X$, and hence by the compactness of $C_{\varphi}: X \longrightarrow X$, some subsequence $\left(f_{n_{k}} \circ \varphi\right)_{k \in \mathbb{N}}$ converges in norm to some function $f \in X$. Again, by the compactness of $C_{\varphi}: X \longrightarrow X$, from the sequence $\left(n_{k}\right)_{k \in \mathbb{N}}$, we may then extract a subsequence $\left(n_{k_{j}}\right)_{j \in \mathbb{N}}$ such that $\left(g_{n_{k_{j}}} \circ \varphi\right)_{j \in \mathbb{N}}$ converges in norm to some $g \in X$. Then, $h:=f+\bar{g} \in X_{H}$ and the sequence $\left(h_{n_{k_{j}}} \circ \varphi\right)_{j \in \mathbb{N}}$ converges in norm to $h$. Therefore, $C_{\varphi}: X_{H} \longrightarrow X_{H}$ is compact.

Lastly, assume $C_{\varphi}: X \longrightarrow X$ is bounded below. Then, there exists some constant $\delta>0$ such that for each $f \in X$, $\|f \circ \varphi\|_{X} \geq \delta\|f\|_{X}$. On the other hand, by (36), there exist constants $C_{1}, C_{2}>0$, such that for each $h=f+\bar{g} \in X_{H}$ with $g(0)=0, C_{1}\left(\|f\|_{X}+\|g\|_{X}\right) \leq\|h\|_{X_{H}} \leq C_{2}\left(\|f\|_{X}+\|g\|_{X}\right)$ Thus,

$$
\begin{aligned}
\|h \circ \varphi\|_{X_{H}} & \geq C_{1}\left(\|f \circ \varphi\|_{X}+\|g \circ \varphi\|_{X}\right) \geq C_{1} \delta\left(\|f\|_{X}+\|g\|_{X}\right) \\
& \geq C_{1} \delta C_{2}\|h\|_{X_{H}},
\end{aligned}
$$

proving that $C_{\varphi}: X_{H} \longrightarrow X_{H}$ is bounded below.

Of course, the conclusion of Theorem 1 also holds if condition (36) holds for the norms of $X$ and $X_{H}$.

Focusing on the compactness of $C_{\varphi}$ acting on the spaces $\mathscr{B}_{H}^{\alpha}, \mathscr{X}_{H}, B_{H}^{p}$, and $\mathrm{BMOA}_{H}$, which satisfy along with the corresponding analytic counterparts $\mathscr{B}^{\alpha}, \mathscr{E}, B^{p}$, and BMOA, the conditions of Theorem 1, from Theorem 2 of [11], Theorem 1.4 of [12], Theorem 4.3 of [13], Theorem 3.7 of [14], Corollary 5 of [15], and Corollary 3.2 and Remark 3.3 of [16] (see also Theorem 1.1 of [17] and Theorem 1 of [18] for alternative characterizations), we deduce the following corollaries. 
Corollary 1. Let $\varphi \in S(\mathbb{D})$ and $\alpha>0$. Then, $C_{\varphi}$ is compact on $\mathscr{B}_{H}^{\alpha}$ if and only if $\varphi \in \mathscr{B}^{\alpha}$ and

$$
\lim _{|\varphi(z)| \longrightarrow 1} \frac{\left(1-|z|^{2}\right)^{\alpha}\left|\varphi^{\prime}(z)\right|}{\left(1-|\varphi(z)|^{2}\right)^{\alpha}}=0 .
$$

Corollary 2. For $\varphi \in S(\mathbb{D})$, a bounded operator $C_{\varphi}$ on $\mathscr{Z}_{H}$ (equivalently on $\mathscr{Z}$ ) is compact if and only if

$$
\begin{array}{r}
\lim _{|\varphi(z)| \longrightarrow 1} \frac{\left(1-|z|^{2}\right)\left|\varphi^{\prime}(z)\right|^{2}}{1-|\varphi(z)|^{2}}=0, \\
\lim _{|\varphi(z)| \longrightarrow 1}\left(1-|z|^{2}\right)\left|\varphi^{\prime \prime}(z)\right| \log \frac{1}{1-|\varphi(z)|^{2}}=0 .
\end{array}
$$

Corollary 3. Let $\varphi \in S(\mathbb{D}), 1<p \leq q, 1<r<q$. If $C_{\varphi}$ is bounded on $B_{H}^{r}$ (equivalently on $B^{r}$ ), then $C_{\varphi}: B_{H}^{p} \longrightarrow B_{H}^{q}$ is compact if and only if $C_{\varphi}: \mathscr{B} \longrightarrow \mathscr{B}$ is compact if and only if

$$
\lim _{|\varphi(z)| \longrightarrow 1} \frac{\left(1-|z|^{2}\right)\left|\varphi^{\prime}(z)\right|}{1-|\varphi(z)|^{2}}=0 .
$$

Corollary 4. For $\varphi \in S(\mathbb{D})$, if $C_{\varphi}$ is bounded on $B_{H}^{1}$ (equivalently on $B^{1}$ ), then $C_{\varphi}: B_{H}^{1} \stackrel{C_{\varphi}}{\longrightarrow} B_{H}^{1}$ is compact if and only if $C_{\varphi}: \mathscr{B} \longrightarrow \mathscr{B}$ is compact if and only if

$$
\lim _{|\varphi(z)| \longrightarrow 1} \frac{\left(1-|z|^{2}\right)\left|\varphi^{\prime}(z)\right|}{1-|\varphi(z)|^{2}}=0 .
$$

Corollary 5. The composition operator $C_{\varphi}$ is compact on $B M O A_{H}$ if and only if

$$
\begin{array}{r}
\lim _{|\varphi(a)| \longrightarrow 1}\left\|L_{\varphi(a)} \circ \varphi \circ L_{a}\right\|_{H^{2}}=0, \text { and, for all } R \in(0,1), \\
\lim _{t \rightarrow 1} \sup _{|\varphi(a)| \leq R} m\left(\left\{\zeta \in \partial \mathbb{D}:\left|\left(\varphi \circ L_{a}\right)(\zeta)\right|>t\right\}\right)=0,
\end{array}
$$

where $m$ is the Lebesgue measure.

Since by [3] and its extension to the corresponding harmonic spaces provided in [4], for $\alpha>0$, the spaces $\mathscr{A}^{-\alpha}$ and $\mathscr{A}_{H}^{-\alpha}$ are equivalent to $\mathscr{B}^{\alpha+1}$ and $\mathscr{B}_{H}^{\alpha+1}$, respectively, and Theorem 1 can be applied to the growth spaces as well. In particular, with the appropriate modification of the parameter, Corollary 1 yields a characterization of compactness on the harmonic growth spaces. Using the following result, which is a special case of Theorem 3.2 of [19], we provide below a simpler characterization.

Theorem 2. Let $\varphi$ be an analytic self-map of $\mathbb{D}, X$ a Banach space of analytic functions with norm $\|\cdot\|$ satisfying the following conditions:

(a) X contains the constants.

(b) For each $z \in \mathbb{D}$, the point-evaluation functional $\delta_{z} f:=f(z)$ is in $X^{*}$. Thus,

$$
\left\|\delta_{z}\right\|=\sup \{|f(z)|: f \in X, f \leq 1\}<\infty .
$$

(c) The unit ball of $X$ is relatively compact with respect to the topology of uniform convergence on compact subsets of $\mathbb{D}$.

(d) There is a constant $C>0$ such that for all $S \in A u t(\mathbb{D})$ and $f \in X$,

$$
\|S f\| \leq C\|f\| .
$$

Let $\alpha>0$ and assume $C_{\varphi}: X \longrightarrow \mathscr{A}^{-\alpha}$ is bounded. Then, $C_{\varphi}: X \longrightarrow \mathscr{A}^{-\alpha}$ is compact if and only if

$$
\lim _{|\varphi(z)| \longrightarrow 1}\left(1-|z|^{2}\right)^{\alpha}\left\|\delta_{\varphi(z)}\right\|=0 .
$$

In the special case, when $X=\mathscr{A}^{-\alpha}$,

$$
|f(z)| \leq \frac{1}{\left(1-|z|^{2}\right)^{\alpha}}\|f\|_{\mathscr{A}^{-\alpha}},
$$

for each $z \in \mathbb{D}$ and $f \in \mathscr{A}^{-\alpha}$. Therefore,

$$
\left\|\delta_{z}\right\| \leq \frac{1}{\left(1-|z|^{2}\right)^{\alpha}} .
$$

Next, note that the constant function 1 has norm 1 in $\mathscr{A}^{-\alpha}$. Thus, in (47), equality holds at $z=0$. On the other hand, for $z=r e^{i \theta}$, for $0<r<1$, and $\theta \in \mathbb{R}$, taking as test function

$$
f_{z}(w)=\frac{1}{\left(1-e^{-2 i \theta} w^{2}\right)^{\alpha}}
$$

we see that $f_{z} \in \mathscr{A}^{-\alpha},\left\|f_{z}\right\|_{\mathscr{A}^{-\alpha}}=1$, and

$$
\frac{1}{\left(1-r^{2}\right)^{\alpha}}=\left|f_{z}(z)\right| \leq\left\|\delta_{z}\right\| \text {. }
$$

Hence,

$$
\left\|\delta_{z}\right\|=\frac{1}{\left(1-|z|^{2}\right)^{\alpha}} .
$$

Since the above conditions (a)-(d) clearly hold for $\mathscr{A}^{-\alpha}$, applying Theorem 2 to $X=\mathscr{A}^{-\alpha}$, using (50), and then taking the ath root, we deduce the following result.

Corollary 6. Let $\varphi \in S(\mathbb{D}), \alpha>0$ and assume $C_{\varphi}$ is bounded on $\mathscr{A}_{H}^{-\alpha}$ (equivalently on $\mathscr{A}^{-\alpha}$ ). Then, $C_{\varphi}$ is compact if and only if

$$
\lim _{|\varphi(z)| \longrightarrow 1} \frac{1-|z|^{2}}{1-|\varphi(z)|^{2}}=0 .
$$

\section{Isometries of $C_{\varphi}$}

In this section, we wish to characterize the isometries on the harmonic spaces $\mathscr{B}_{H}^{\alpha}, \mathscr{A}_{H}^{-\alpha}, \mathscr{X}_{H}$, and $B_{H}^{p}$. For most of the corresponding analytic counterparts, namely, $\mathscr{B}^{\alpha}$ for $\alpha \neq 1$, $\mathscr{A}^{-\alpha}, \mathscr{Z}$, and $B^{p}$ for $p>1$ and $p \neq 2$, the only composition operators that are isometries are those induced by rotations [20-23] (see also [24]). Since an isometry on a harmonic space $X_{H}$ that extends an analytic space $X$ is also an isometry on $X$, $C_{\varphi}$ is an isometry on $\mathscr{B}_{H}^{\alpha}$ for $\alpha \neq 1, \mathscr{A}_{H}^{-\alpha}$ for $\alpha>0, \mathscr{Z}_{H}$ and $B_{H}^{p}$ 
for $p>1, p \neq 2$, if and only if $\varphi$ is a rotation. In the case of the minimal Möbius-invariant space $B^{1}$ under the Möbiusinvariant norm (18), composition operators induced by a disk automorphism are clearly isometries. In fact, Bao and Wulan proved in Theorem 5.2 of [25] that the isometric isomorphisms on $B^{1}$ are all of the form $\lambda C_{\varphi}$, where $\lambda$ is a unimodular constant and $\varphi \in A u t(\mathbb{D})$. Of course, these are also isometries when regarding $C_{\varphi}$ as an operator on $B_{H}^{1}$. To the best of our knowledge, it is not known whether linear isometries among the composition operators on $B^{1}$ other than those induced by disk automorphisms exist. The following result partially answers this question.

Theorem 3. If $\varphi \in S(\mathbb{D})$ has a fixed point in $\mathbb{D}$, then $C_{\varphi}: B^{1} \longrightarrow B^{1}$ is an isometry if and only if $\varphi \in A u t(\mathbb{D})$.

Proof. The sufficiency is clear. Assume $p \in \mathbb{D}$ is a fixed point of $\varphi, C_{\varphi}: B^{1} \longrightarrow B^{1}$ is an isometry, and $\varphi$ is not a disk automorphism. First, assume $p=0$. Then, $\varphi$ is not a rotation, and the $n$th iterate $\varphi_{n}$ of $\varphi$ is also an isometry of $B^{1}$ for any $n \in \mathbb{N}$. By the Grand Iteration Theorem (see [26], p. 78), the sequence $\left(\varphi_{n}\right)$ converges to 0 uniformly on compact subsets of $\mathbb{D}$. Thus, $\lim _{n \rightarrow \infty}\left\|\varphi_{n}\right\|_{\infty}=0$. Therefore, for all $n$ sufficiently large, $\left\|\varphi_{n}\right\|_{\infty}<1 / 2$. Hence, for such an $n, C_{\varphi_{n}}$ is a compact operator on $\mathscr{B}$, and by Corollary 4 , it is also compact on $B^{1}$. By Lemma 3.7 of [14], since the sequence $\left(p_{k}\right)_{k=2}^{\infty}$ defined by $p_{k}(z)=(1 /(k-1)) z^{k}$ is bounded in $B^{1}$ and converges to 0 uniformly on compact subsets of $\mathbb{D}$,

$$
\lim _{k \longrightarrow \infty}\left\|C_{\varphi_{n}} p_{k}\right\|_{B^{1}}=0 \text {. }
$$

On the other hand, since $\varphi_{n}$ is an isometry of $B^{1}$, $\left\|C_{\varphi_{n}} p_{k}\right\|_{B^{1}}=\left\|p_{k}\right\|_{B^{1}}$ is bounded away from 0 , as due to the equivalence of the two norms on $B^{1}$,

$$
\left\|p_{k}\right\|=\int_{\mathbb{D}}\left|p_{k}^{\prime \prime}(z)\right| \mathrm{d} A(z)=2 \int_{0}^{1} k r^{k-1} \mathrm{~d} r=2 .
$$

We have reached a contradiction. Therefore, $\varphi$ must be a rotation in this case.

Next, assume $\varphi(p)=p$ for some $p \in \mathbb{D}$. Due to the Möbius invariance of $\|\cdot\|_{B^{1}}$, letting $L_{p}$ denote the disk automorphism interchanging 0 and $p$, the function $\psi=L_{p} \circ \varphi \circ L_{p}$ induces an isometry on $B^{1}$ and has 0 as a fixed point. By the previous case, $\psi$ must be a rotation. Then, $\varphi=$ $L_{p} \circ \psi \circ L_{p}$ is a disk automorphism.

We conjecture that the conclusion of Theorem 3 also holds if $\varphi$ does not have fixed points in $\mathbb{D}$. In this case, if $\varphi \notin A u t(\mathbb{D})$, then $\varphi$ has a boundary fixed point $\omega$ (i.e., the Denjoy-Wolff point of $\varphi$ ) such that $\varphi_{n} \longrightarrow \omega$ locally uniformly, and $\varphi$ has an angular derivative at $\omega$ (see [26], p. 78).

We shall see that by contrast, the Bloch space has a very rich set of isometries among the composition operators. This feature is shared by the space BMOA with respect to the Möbius-invariant seminorm. As noted in Proposition 2.1 of [27], all such isometries must be induced by a symbol that fixes 0. Laitila observed in Corollary 2 of [27] that the isometries $C_{\varphi}$ on the Bloch space are also isometries on BMOA. However, the inclusion is proper. For example, finite Blaschke products induce isometries $C_{\varphi}$ on BMOA but not on $\mathscr{B}$ unless they are rotations. We are not aware of results of this type on BMOA with respect to the equivalent norm $\|\cdot\|_{*}$. Thus, we leave to the reader the following problem for future investigation.

Open questions:

(1) Are there any isometries among the composition operators on BMOA with respect to the norm $\|\cdot\|_{*}$ other than those induced by rotations?

(2) If this question has a positive answer, are all nontrivial isometries on BMOA also isometries on $\mathrm{BMOA}_{H}$ ?

To complete the study of the isometries on the harmonic spaces under consideration in our work, it remains to analyze the cases when $C_{\varphi}$ acts on the harmonic Bloch space $\mathscr{B}_{H}$ and on the harmonic Dirichlet space $\mathscr{D}_{H}$ to determine whether the nontrivial isometries $C_{\varphi}$ on $\mathscr{B}$ and $\mathscr{D}$ are also isometries on the larger spaces $\mathscr{B}_{H}$ and $\mathscr{D}_{H}$. We shall prove that this is indeed the case.

We shall make use of the following results.

Theorem 4 (see [28], Theorem 5 and Corollary 2). Let $\varphi: \mathbb{D} \longrightarrow \mathbb{D}$ analytic function, then the following are equivalent:

(a) $C_{\varphi}: \mathscr{B} \longrightarrow \mathscr{B}$ is an isometry.

(b) $\varphi(0)=0$ and $\|\varphi\|_{\mathscr{B}}=1$.

(c) Either $\varphi(z)=\lambda z$ for some $\lambda \in \mathbb{C},|\lambda|=1$, or $\varphi=g B$, where $g \in S(\mathbb{D})$ is nonvanishing and $B$ is an infinite Blaschke product whose zeros form a sequence $\left(z_{n}\right)_{n \in \mathbb{N}}$ containing 0 and a subsequence $\left(z_{n_{j}}\right)_{j \in \mathbb{N}}$ such that $\left|g\left(z_{n_{j}}\right)\right| \longrightarrow 1$ and

$$
\lim _{j \longrightarrow \infty} \prod_{k \neq n_{j}}\left|\frac{z_{n_{j}}-z_{k}}{1-\overline{z_{n_{j}}} z_{k}}\right|=1 \text {. }
$$

Theorem 5 (see [29], Theorem 2.7). For $\varphi \in S(\mathbb{D}), C_{\varphi}$ is an isometry on $\mathscr{B}$ if and only if $\varphi(0)=0$ and either $\varphi$ is a rotation or for every $a \in \mathbb{D}$ there exists a sequence $\left(z_{n}\right)$ in $\mathbb{D}$ such that $\left|z_{n}\right| \longrightarrow 1, \varphi\left(z_{n}\right)=a$ and

$$
\lim _{n \longrightarrow \infty}\left(1-\left|z_{n}\right|^{2}\right)\left|\varphi^{\prime}\left(z_{n}\right)\right|=1-|a|^{2} \text {. }
$$

We can now prove our main result in this section.

Theorem 6. Let $\varphi$ be an analytic self-map of $\mathbb{D}$. Then, the following statements are equivalent.

(a) $C_{\varphi}$ is an isometry on $\mathscr{B}_{H}$.

(b) $C_{\varphi}$ is an isometry on $\mathscr{B}$.

(c) $\varphi(0)=0$ and $\varphi_{\mathscr{B}}=1$.

Proof. Since by Theorem 4, (b) is equivalent to (c), and as observed above, (a) clearly implies (b); we only need to show that (c) implies (a).

Assume $\varphi(0)=0$ and $\|\varphi\|_{\mathscr{B}}=1$. Then, $\left\|C_{\varphi}\right\|=1$. If $\varphi$ is a rotation, the result is clear. So, assume $\varphi$ is not a rotation. 
Observe that since $\varphi(0)=0$, it suffices to show that $C_{\varphi}$ is seminorm preserving on $\mathscr{B}_{H}$. Equivalently, $\|h \circ \varphi\|_{\mathscr{B}_{H}}=$ $\|h\|_{\mathscr{B}_{H}}$ for all $h \in \mathscr{B}_{H}$ such that $h(0)=0$. Also, by dividing by the norm, we only need to prove that $\|h \circ \varphi\|_{\mathscr{B}_{H}}=1$ for $\|h\|_{\mathscr{B}_{H}}=1$ with $h(0)=0$.

So, assume $h$ satisfies the conditions $\|h\|_{\mathscr{B}_{H}}=1$ and $h(0)=0$. Then,

$$
\sup _{z \in \mathbb{D}}\left(1-|z|^{2}\right)\left(\left|h_{z}(z)\right|+\left|h_{\bar{z}}(z)\right|\right)=1 .
$$

Thus, either this supremum is attained at some point inside the disk or it is a limit along a sequence of points in $\mathbb{D}$ approaching the unit circle. Specifically, one of the following two cases must hold:

(i) There exists $a \in \mathbb{D}$ such that

$$
\left(1-|a|^{2}\right)\left(\left|h_{z}(a)\right|+\left|h_{\bar{z}}(a)\right|\right)=1 \text {. }
$$

(ii) There exists a sequence $\left(a_{k}\right)$ is $\mathbb{D}$ such that $\left|a_{k}\right| \longrightarrow 1$ and

$$
\lim _{k \rightarrow \infty}\left(1-\left|a_{k}\right|^{2}\right)\left(\left|h_{z}\left(a_{k}\right)\right|+\left|h_{\bar{z}}\left(a_{k}\right)\right|\right)=1 .
$$

Assume first (i) holds. Then, by Theorem 5, there exists a sequence $\left(z_{n}\right)$ in $\mathbb{D}$ such that $\left|z_{n}\right| \longrightarrow 1$ as $n \longrightarrow \infty, \varphi\left(z_{n}\right)=$ $a$ for each $n \in \mathbb{N}$, and

$$
\lim _{n \longrightarrow \infty}\left(1-\left|z_{n}\right|^{2}\right)\left|\varphi^{\prime}\left(z_{n}\right)\right|=1-|a|^{2} .
$$

Therefore,

$$
\begin{aligned}
& \left(1-\left|z_{n}\right|^{2}\right)\left(\left|(h \circ \varphi)_{z}\left(z_{n}\right)\right|+\left|(h \circ \varphi)_{\bar{z}}\left(z_{n}\right)\right|\right) \\
& =\left(1-\left|z_{n}\right|^{2}\right)\left(\left|h_{z}\left(\varphi\left(z_{n}\right)\right) \varphi^{\prime}\left(z_{n}\right)\right|+\left|h_{\bar{z}}\left(\varphi\left(z_{n}\right)\right) \overline{\varphi^{\prime}\left(z_{n}\right)}\right|\right) \\
& =\left(1-\left|z_{n}\right|^{2}\right)\left|\varphi^{\prime}\left(z_{n}\right)\right|\left(\left|h_{z}(a)\right|+\left|h_{\bar{z}}(a)\right|\right) \\
& \longrightarrow\left(1-|a|^{2}\right)\left(\left|h_{z}(a)\right|+\left|h_{\bar{z}}(a)\right|\right), \quad \text { as } n \longrightarrow \infty .
\end{aligned}
$$

Using (i), it follows that

$$
\begin{aligned}
1 & =\lim _{n \longrightarrow \infty}\left(1-\left|z_{n}\right|^{2}\right)\left(\left|(h \circ \varphi)_{z}\left(z_{n}\right)\right|+\left|(h \circ \varphi)_{\bar{z}}\left(z_{n}\right)\right|\right) \\
& \leq \sup _{z \in \mathbb{D}}\left(1-|z|^{2}\right)\left(\left|(h \circ \varphi)_{z}(z)\right|+\left|(h \circ \varphi)_{\bar{z}}(z)\right|\right) \\
& =\left\|C_{\varphi} h\right\|_{\mathscr{B}_{H}} .
\end{aligned}
$$

Since, as observed above, the operator $C_{\varphi}$ has norm 1 , $\left\|C_{\varphi} h\right\|_{\mathscr{B}_{H}} \leq 1$. It follows that $\left\|C_{\varphi} h\right\|_{\mathscr{B}_{H}}=1$.

Next, assume (ii) holds. Then, again by Theorem 5 , for each $k \in \mathbb{N}$, there exists a sequence $\left(z_{n, k}\right)_{n \in \mathbb{N}}$ in $\mathbb{D}$ such that $\left|z_{n, k}\right| \longrightarrow 1$ as $n \longrightarrow \infty, \varphi\left(z_{n, k}\right)=a_{k}$ and

$$
\lim _{n \longrightarrow \infty}\left(1-\left|z_{n, k}\right|^{2}\right)\left|\varphi^{\prime}\left(z_{n, k}\right)\right|=1-\left|a_{k}\right|^{2} .
$$

Proceeding as above, for each $k \in \mathbb{N}$, we have

$$
\begin{aligned}
& \left(1-\left|z_{n, k}\right|^{2}\right)\left(\left|(h \circ \varphi)_{z}\left(z_{n, k}\right)\right|+\left|(h \circ \varphi)_{\bar{z}}\left(z_{n, k}\right)\right|\right) \\
& =\left(1-\left|z_{n, k}\right|^{2}\right)\left|\varphi^{\prime}\left(z_{n, k}\right)\right|\left(\left|h_{z}\left(a_{k}\right)\right|+\left|h_{\bar{z}}\left(a_{k}\right)\right|\right) \\
& \longrightarrow\left(1-\left|a_{k}\right|^{2}\right)\left(\left|h_{z}\left(a_{k}\right)\right|+\left|h_{\bar{z}}\left(a_{k}\right)\right|\right), \quad \text { as } n \longrightarrow \infty .
\end{aligned}
$$

Hence, taking the limit as $k \longrightarrow \infty$ and using (ii), we deduce that

$$
\begin{aligned}
1 & =\lim _{k \longrightarrow \infty}\left(1-\left|a_{k}\right|^{2}\right)\left(\left|h_{z}\left(a_{k}\right)\right|+\left|h_{\bar{z}}\left(a_{k}\right)\right|\right) \\
& =\lim _{k \longrightarrow \infty} \lim _{n \longrightarrow \infty}\left(1-\left|z_{n, k}\right|^{2}\right)\left(\left|(h \circ \varphi)_{z}\left(z_{n, k}\right)\right|+\left|(h \circ \varphi)_{\bar{z}}\left(z_{n, k}\right)\right|\right) \\
& \leq \sup _{z \in \mathbb{D}}\left(1-|z|^{2}\right)\left(\left|(h \circ \varphi)_{z}(z)\right|+\left|(h \circ \varphi)_{\bar{z}}(z)\right|\right) \\
& =\left\|C_{\varphi} h\right\|_{\mathscr{B}_{H}} .
\end{aligned}
$$

Thus, $\left\|C_{\varphi} h\right\|_{\mathscr{B}_{H}}=1$ also in this case. Therefore, $C_{\varphi}$ is an isometry on $\mathscr{B}_{H}$.

We now turn our attention to the identification of the isometries on $\mathscr{D}_{H}$.

A function $\varphi \in S(\mathbb{D})$ is said to be a univalent full map if it is one-to-one, and the complement of the range of $\varphi$ has null area measure. In [30], the authors characterize the isometries among the composition operators on the Dirichlet space $\mathscr{D}$.

Theorem 7 (see [30], p.1703). A composition operator $C_{\varphi}$ acting on the Dirichlet space $\mathscr{D}$ is an isometry if and only if $\varphi$ is a univalent full map of $\mathbb{D}$ that fixes 0 .

We are ready to characterize the isometry on $\mathscr{D}_{H}$.

Theorem 8. The bounded composition operator $C_{\varphi}$ on $\mathscr{D}_{H}$ is an isometry if and only if $\varphi$ is a univalent full map fixing 0 .

Proof. Assume $C_{\varphi}$ is an isometry on $\mathscr{D}_{H}$. Since the Dirichlet space $\mathscr{D}$ is a subspace of $\mathscr{D}_{H}$ and the norm of $\mathscr{D}_{H}$ equals the norm of $\mathscr{D}$, the operator $C_{\varphi}$ is also an isometry on $\mathscr{D}$. Thus, by Theorem 7, $\varphi$ is a univalent full map fixing the origin.

Conversely, assume $\varphi$ is a univalent full map fixing the origin. Let $h \in \mathscr{D}_{H}$. Then, $h=f+\bar{g}$ with $f, g \in H(\mathbb{D})$ and $g(0)=0$. So, $f, g \in \mathscr{D}$, and

$$
C_{\varphi} h=C_{\varphi} f+C_{\varphi} \bar{g}=C_{\varphi} f+\overline{C_{\varphi} g} .
$$

Thus, by Theorem 7, $\left\|C_{\varphi} f\right\|_{\mathscr{D}}=\|f\|_{\mathscr{D}},\left\|C_{\varphi} g\right\|_{\mathscr{D}}=\|g\|_{\mathscr{D}}$. Therefore, by (15), we have

$$
\begin{aligned}
\left\|C_{\varphi} h\right\|_{\mathscr{D}_{H}}^{2} & =\left\|C_{\varphi} f\right\|_{\mathscr{D}}^{2}+\left\|C_{\varphi} g\right\|_{\mathscr{D}}^{2} \\
& =\|f\|_{\mathscr{D}}^{2}+\|g\|_{\mathscr{D}}^{2} \\
& =\|h\|_{\mathscr{D}_{H}}^{2},
\end{aligned}
$$

proving that $C_{\varphi}$ is an isometry on $\mathscr{D}_{H}$. 


\section{Eigenfunctions of $C_{\varphi}$}

In the early 1870s, Ernst Schröder introduced the eigenvalue equation for composition operators. Suppose $\varphi$ is an analytic self-map of $\mathbb{D}$, and $\lambda$ is complex constant. The functional equation

$$
f \circ \varphi=\lambda f, \quad \text { for } f \in H(\mathbb{D}),
$$

is known as Schröder's equation.

Lemma 1. Assume $\varphi \in S(\mathbb{D}), \varphi$ nonconstant, $p \in \mathbb{D}$ with $\varphi(p)=p$. Let $\lambda \neq 1$, and suppose $f$ is an analytic function such that for each $z \in \mathbb{D}$

$$
f \circ \varphi(z)=\lambda f(z)
$$

$$
\text { If } \varphi^{\prime}(p)=0 \text {, then } f \equiv 0 \text {. }
$$

Proof. Assume an analytic function $f$ satisfying equation (68) exists with $f$ not identically zero. Since $\varphi(p)=p$ and $\varphi^{\prime}(p)=0$, there is a natural number $k>1$, such that for $z$ sufficiently near $p$

$$
\varphi(z)-p=(z-p)^{k} \psi(z)
$$

for some analytic function $\psi$ with $\psi(p) \neq 0$. Evaluating equation (68) at $p$, we get

$$
f(p)=f(\varphi(p))=\lambda f(p) .
$$

Since $\lambda \neq 1$, it follows that $f(p)=0$. Thus, in some neighborhood of $p, f$ admits the representation of the form

$$
f(z)=(z-p)^{m} g(z),
$$

for some analytic function $g$ such that $g(p) \neq 0$ and $m \in \mathbb{N}$. Then, substituting this expression into equation (68), we obtain

$$
\begin{aligned}
(z-p)^{k m} \psi(z)^{m} g(\varphi(z)) & =(\varphi(z)-p)^{m} g(\varphi(z)) \\
& =\lambda(z-p)^{m} g(z),
\end{aligned}
$$

for $z$ near $p$. Since $k m>m$, we have reached a contradiction.

Lemma 2. Assume $\varphi \in S(\mathbb{D})$ fixes 0 and that $\varphi$ is not a rotation. Then, the only eigenfunctions of $C_{\varphi}$ relative to the eigenvalue 1 are the nonzero constants.

Proof. Assume 1 is an eigenvalue of $C_{\varphi}$, and let $f$ be a corresponding eigenfunction. Then, $f \circ \varphi=f$ implies that for all $n \in \mathbb{N}$

$$
f \circ \varphi_{n}=f,
$$

where $\varphi_{n}$ is the $n^{\text {th }}$ iterate of $\varphi$.

Fix $a \in \mathbb{D}, a \neq 0$. Let $a_{n}=\varphi_{n}(a)$ for all $n \in \mathbb{N}$. Then, the sequence $\left(a_{n}\right)$ must converge to 0 . Thus,

$$
f\left(a_{n}\right)=f\left(\varphi_{n}(a)\right)=f(a), \quad \text { for all } n \in \mathbb{N} .
$$

By continuity $f(a)=f(0)$. Therefore, $f$ is constant.

For $\varphi \in S(\mathbb{D})$ which fixes a point $p \in \mathbb{D}$ and such that $0<\left|\varphi^{\prime}(p)\right|<1$, the unique solution $\sigma$ of Schröder's equation corresponding to the eigenvalue $\varphi^{\prime}(p)$ such that $\sigma^{\prime}(p)=1$ is called the Königs function of $\varphi$ or principal eigenfunction of $C_{\varphi}$.

We state below Königs' theorem, which gives a description of the eigenvalues and corresponding eigenfunctions of the composition operator $C_{\varphi}$, considered as a linear transformation on $H(\mathbb{D})$, when the symbol is nonautomorphic with a fixed point in $\mathbb{D}$.

Theorem 9 (see [26]). Let $\varphi \in S(\mathbb{D}) \backslash A$ ut $(\mathbb{D})$ with a fixed point $p \in \mathbb{D}$.

(a) If $\varphi^{\prime}(p)=0$, then 1 is the only eigenvalue of $C_{\varphi}$.

(b) If $\varphi^{\prime}(p) \neq 0$, then the set of eigenvalues of $C_{\varphi}$ is given by

$$
\left\{\varphi^{\prime}(p)^{n}: n \in \mathbb{N} \cup\{0\}\right\}
$$

Each eigenvalue has multiplicity 1 , and the function $\sigma^{n}$ spans the eigenspace for $\varphi^{\prime}(p)^{n}(n \in \mathbb{N})$.

(c) If $\varphi$ is univalent, then so is $\sigma$.

The following result is a special case of a theorem proved by Hammond in his doctoral dissertation that extends to a general Banach space of analytic functions on $\mathbb{D}$ the Eigenfunction Theorem valid for the Hardy Hilbert space (see [26], p.94).

Theorem 10 (see [31]). If $C_{\varphi}$ is compact on a Banach space $X$ of analytic functions on $\mathbb{D}$, then the eigenfunctions $\sigma^{n}$ belong to the space $X$ for each $n \in \mathbb{N}$.

Using Theorem 10, Paudyal in [32] obtained the following sufficient condition that ensures that all the powers of the Königs function belong to the $\alpha$-Bloch space.

Theorem 11. (see [32], Theorem 2.1.9). Suppose $\varphi \in S(\mathbb{D})$ with $\varphi(0)=0,0<\left|\varphi^{\prime}(0)\right|<1, C_{\varphi}$ is bounded on $\mathscr{B}^{\alpha}$ for some $\alpha>0$, and there exists $k \in \mathbb{N}$ such that $\left\|\varphi_{k}\right\|_{\infty}<1$. Then, $\sigma^{n} \in \mathscr{B}^{\alpha}$ for all $n \in \mathbb{N}$.

Since for $\alpha>0$, the growth space $\mathscr{A}^{-\alpha}$ can be identified with the Bloch-type space $\mathscr{B}^{\alpha+1}$, the conclusion of Theorem 11 also holds when $C_{\varphi}$ is bounded on $\mathscr{A}^{-\alpha}$. Arguing as in the proof of Theorem 11 provided in [32] since composition operators whose symbol has supremum norm smaller than 1 are compact on the Zygmund space, the Besov spaces, and BMOA, using Theorem 10, we obtain the following extension to the other spaces treated in this paper.

Corollary 7. Suppose $\varphi \in S(\mathbb{D})$ with $\varphi(0)=0$, $0<\left|\varphi^{\prime}(0)\right|<1, C_{\varphi}$ is bounded on $\mathscr{Z}$, respectively, on $B^{p}$ for $p \geq 1$, respectively, on $B M O A$, and there exists $k \in \mathbb{N}$ such that $\left\|\varphi_{k}\right\|_{\infty}<1$. Then, $\sigma^{n} \in \mathscr{Z}$, respectively, $B^{p}$, respectively, $B M O A$, for all $n \in \mathbb{N}$.

As observed above, if $\varphi \in S(\mathbb{D})$ with $\varphi(0)=0$ and $0<\left|\varphi^{\prime}(0)\right|<1$, then the only eigenvalues of $C_{\varphi}$ must be of the form $\varphi^{\prime}(0)^{n}$ for some $n \in \mathbb{N} \cup\{0\}$, and the corresponding eigenfunctions must be constant multiples of $\sigma^{n}$. 
We are now interested in determining the eigenfunctions of $C_{\varphi}$ as an operator acting on spaces of harmonic mappings with domain $\mathbb{D}$. The following result shows that these eigenfunctions are closely related to the eigenfunctions of the analytic counterparts.

Theorem 12. Let $\varphi \in S(\mathbb{D})$ with $\varphi(0)=0$ and $0<\left|\varphi^{\prime}(0)\right|<1$. Suppose $\lambda \in \mathbb{C}$ and $h$ is a harmonic mapping on $\mathbb{D}$ such that

$$
h \circ \varphi=\lambda h .
$$

Then, either $\lambda=1$, in which case $h$ is constant, or there exists $n \in \mathbb{N}$ such that $\lambda=\varphi^{\prime}(0)^{n}$. In the latter case, $h$ is a linear combination of $\sigma^{n}$ and its conjugate. Moreover, the argument of $\lambda$ must be a rational multiple of $\pi$. When reduced to lowest terms, the denominator of this rational factor of $\pi$ must be a divisor of $n$.

Proof. Let $f, g \in H(\mathbb{D})$ with $g(0)=0$ and $h=f+\bar{g}$. Then, by (76),

$$
f \circ \varphi+\overline{g \circ \varphi}=\lambda(f+\bar{g})
$$

so rearranging the terms, we obtain

$$
f \circ \varphi-\lambda f=\lambda \bar{g}-\overline{g \circ \varphi} .
$$

Since the left-hand side of (78) is analytic, and the righthand side is antianalytic, their common value must be a constant $\eta$. Thus,

$$
\begin{aligned}
& f \circ \varphi-\lambda f=\eta, \\
& \overline{g \circ \varphi}-\lambda \bar{g}=\eta .
\end{aligned}
$$
Hence,

Since $\varphi(0)=0$ and $g(0)=0$, from (79), we obtain $\eta=0$.

$$
\begin{aligned}
& f \circ \varphi=\lambda f, \\
& \overline{g \circ \varphi}=\lambda \bar{g} .
\end{aligned}
$$

If $\lambda=1$, then by Lemma 2, the only analytic functions $F$ satisfying the equation $F \circ \varphi=F$ are the constant functions. Thus, $g$ is the constant 0 , and $h=f$ is constant.

Next assume $\lambda \neq 1$ and $\varphi^{\prime}(0)=r e^{i \theta}$ with $0<r<1$ and $\theta \in \mathbb{R}$. From Königs' Theorem applied to the equation $f \circ \varphi=\lambda f$, it follows that $\lambda$ must be a power of $\varphi^{\prime}(0)$, and $f$ is a multiple of the corresponding power of $\sigma$, that is, there exist an $n \in \mathbb{N}$ and $\alpha \in \mathbb{C}$ such that $\lambda=\varphi^{\prime}(0)^{n}$ and $f=\alpha \sigma^{n}$.

On the other hand, $g \circ \varphi=\bar{\lambda} g$, so, again by Königs' Theorem, there exist $m \in \mathbb{N}$ and $\beta \in \mathbb{C}$ such that $\bar{\lambda}=\varphi^{\prime}(0)^{m}$ and $g=\beta \sigma^{m}$. Then, $r^{n} e^{i n \theta}=r^{m} e^{-i m \theta}$. Thus, $n=m$ and $e^{2 i n \theta}=1$. Therefore,

$$
h=\alpha \sigma^{n}+\bar{\beta} \bar{\sigma}^{n}
$$

and $\theta=\pi k / n$, for some integer $k$, completing the proof.

Remark 1. From the above, we see that if $h$ satisfies (76), where $\lambda=\varphi^{\prime}(0)$ (which corresponds to the special case $n=1)$, then $\varphi^{\prime}(0)$ must be real.
In the context of the composition operator acting on the harmonic spaces $X_{H}$ treated in this work, equivalently, on $X=X_{H} \cap H(\mathbb{D})$, a natural question that arises is as follows.

For which symbols $\varphi$ fixing 0 is the set of eigenvalues of $C_{\varphi}$ regarded as an operator on $X_{H}$ given by $\left\{\varphi^{\prime}(0)^{n}\right.$ : $n \in \mathbb{N} \cup\{0\}\}$ ?

By Theorem 12, the required conditions are that $\varphi^{\prime}(0)$ must be real, and all the powers of the Königs function $\sigma$ must be in $X$.

In particular, every compact operator $C_{\varphi}$ on $X$ (equivalently, on $X_{H}$ ) satisfies the condition $\sigma^{n} \in X$ for all $n \in \mathbb{N}$. Since $C_{\varphi}$ is a compact operator whenever $\|\varphi\|_{\infty}<1$, we are interested in the case when the closure of the range of $\varphi$ intersects the unit circle.

As a consequence of Theorem 11 and Corollary 7, we can now give a simple example of a nonunivalent function $\varphi \in S(\mathbb{D})$ with supremum norm 1 for which the operator $C_{\varphi}: X \longrightarrow X$ is noncompact and has $\left\{\varphi^{\prime}(0)^{n}: n \in \mathbb{N} \cup\{0\}\right\}$ as full set of eigenvalues for any of the spaces $X$ treated in this work.

Example: consider the polynomial:

$$
\varphi(z)=\frac{1}{2}\left(z^{2}-z\right) .
$$

Then, $\varphi$ maps $\mathbb{D}$ into itself, has 0 as a fixed point, $\varphi^{\prime}(0)=$ $-1 / 2$ and is not univalent since $\varphi(1 / 4)=\varphi(3 / 4)$. Moreover, regarding $\varphi$ as a function on $\mathbb{D},\|\varphi\|_{\infty}=1$ since $\varphi(-1)=1$. In fact, $\varphi^{-1}(1)=\{-1\}$, and a straightforward computation shows that $\varphi(\overline{\mathbb{D}})$ touches the unit circle only at the point 1 .

Let us first focus on the case when $X$ is $\mathscr{B}^{\alpha}$. Since

$$
\sup _{z \in \mathbb{D}} \frac{\left(1-|z|^{2}\right)^{\alpha}\left|\varphi^{\prime}(z)\right|}{\left(1-|\varphi(z)|^{2}\right)^{\alpha}}<\infty,
$$

by Theorem 1.4 of [12], the operator $C_{\varphi}$ is bounded on $\mathscr{B}^{\alpha}$ or $\mathscr{B}_{H}^{\alpha}$. Moreover, for $z \in(-1,0)$,

$$
\begin{aligned}
1-\frac{1}{4}\left|z^{2}-z\right|^{2} & =\frac{1}{4}\left(4-|z|^{2}|z-1|^{2}\right) \\
& =\frac{1}{4}(2+|z|)(1-|z|)\left(2+|z|+|z|^{2}\right) .
\end{aligned}
$$

Thus, as $z \longrightarrow-1^{+}$along the real axis,

$\frac{\left(1-|z|^{2}\right)^{\alpha}(1 / 2)|2 z-1|}{\left(1-(1 / 4)\left|z^{2}-z\right|^{2}\right)^{\alpha}}=\frac{2^{2 \alpha-1}(1+|z|)^{\alpha}|2 z-1|}{(2+|z|)^{\alpha}\left(2+|z|+|z|^{2}\right)^{\alpha}} \longrightarrow\left(\frac{2}{3}\right)^{\alpha-1}$.

Hence,

$\lim _{|\varphi(z)| \longrightarrow 1} \frac{\left(1-|z|^{2}\right)^{\alpha}\left|\varphi^{\prime}(z)\right|}{\left(1-|\varphi(z)|^{2}\right)^{\alpha}}=\lim _{z \longrightarrow-1^{+}} \frac{\left(1-|z|^{2}\right)^{\alpha}(1 / 2)|2 z-1|}{\left(1-(1 / 4)\left|z^{2}-z\right|^{2}\right)^{\alpha}} \neq 0$,

where the last limit is taken along the real axis.

Therefore, by Corollary 1 , the operator $C_{\varphi}$ is not compact on $\mathscr{B}^{\alpha}$ or $\mathscr{B}_{H}^{\alpha}$.

Since the only point of contact of $\varphi$ with the unit circle occurs at -1 and $\varphi_{2}(-1)=\varphi(1)=0$, we see that $\left\|\varphi_{2}\right\|_{\infty}<1$. Thus, by Theorem $11, \sigma^{n} \in \mathscr{B}^{\alpha}$ for all $n \in \mathbb{N}$. 
Of course, the same conclusion holds for the growth spaces $\mathscr{A}^{-\alpha}$ since, as observed in the paragraph before Theorem 2, they are isomorphic to the $\mathscr{B}^{\alpha+1}$.

Let $X$ be the Zygmund space. Then, by Theorem 3.1 of [13], the conditions that guarantee the boundedness of $C_{\varphi}$ as an operator on $\mathscr{Z}$ (or $\mathscr{Z}_{H}$ ) are

$$
\begin{array}{r}
\sup _{z \in \mathbb{D}} \frac{\left(1-|z|^{2}\right)\left|\varphi^{\prime}(z)\right|^{2}}{1-|\varphi(z)|^{2}}<\infty, \\
\sup _{z \in \mathbb{D}}\left(1-|z|^{2}\right)\left|\varphi^{\prime \prime}(z)\right| \log \frac{1}{1-|\varphi(z)|^{2}}<\infty,
\end{array}
$$

which are both satisfied by the polynomial $\varphi$. By Theorem 2, $C_{\varphi}$ is not compact on $\mathscr{Z}$ or $\mathscr{Z}_{H}$ since

$$
\begin{aligned}
\lim _{|\varphi(z)| \longrightarrow 1} \frac{\left(1-|z|^{2}\right)\left|\varphi^{\prime}(z)\right|^{2}}{1-|\varphi(z)|^{2}} & =\lim _{z \longrightarrow-1^{+}} \frac{\left(1-|z|^{2}\right)(1 / 4)|2 z-1|^{2}}{1-(1 / 4)\left|z^{2}-z\right|^{2}} \\
& =\lim _{z \longrightarrow-1^{+}} \frac{(1+|z|)(2|z|+1)^{2}}{(2+|z|)\left(2+|z|+|z|^{2}\right)}=\frac{3}{2},
\end{aligned}
$$

where the last two limits are taken along the real axis.

By Corollary $7, \sigma^{n} \in \mathscr{Z}$ for each $n \in \mathbb{N}$.

Assume next $X$ is the Besov space $B^{p}$. By Theorem 18 of [9], since $\varphi^{\prime \prime}$ is constant, $C_{\varphi}$ is bounded as an operator on the minimal Möbius-invariant space $B^{1}$. Since the Besov spaces $B^{P}$ are interpolating spaces between $B^{1}$ and $\mathscr{B}$ and $C_{\varphi}$ is bounded on $\mathscr{B}, C_{\varphi}$ is bounded on $B^{p}$ for any $p>1$. By Corollaries 3.3 and 3.4 , and having shown above that $C_{\varphi}$ is not compact on $\mathscr{B}$, this operator is not compact on $B^{p}$ either. By Corollary $7, \sigma^{n} \in B^{p}$ for each $n \in \mathbb{N}$ and $p \geq 1$.

Finally, assume $X$ is the space BMOA. It is well known that every composition operator on BMOA is bounded. Thus, by Corollary $7, \sigma^{n} \in \mathrm{BMOA}$ for each $n \in \mathbb{N}$. By Theorem 4.1 of [33], if $C_{\varphi}: \mathrm{BMOA} \longrightarrow \mathrm{BMOA}$ is compact, then $C_{\varphi}: H^{2} \longrightarrow H^{2}$. On the other hand, if $\varphi$ was the symbol of a compact composition operator on $\mathrm{H}^{2}$, then (see [26], p. 43)

$$
\lim _{|z| \longrightarrow 1} \frac{1-|\varphi(z)|}{1-|z|}=\infty .
$$

However, the above calculations show that

$$
\begin{aligned}
\lim _{|z| \longrightarrow 1} \frac{1-|\varphi(z)|}{1-|z|} & \leq \lim _{|z| \longrightarrow 1} \frac{1-|\varphi(z)|^{2}}{1-|z|} \\
& =\lim _{r \longrightarrow 1^{-}}\left(r^{2}+r+2\right)(r+2)=12 .
\end{aligned}
$$

Thus, $C_{\varphi}$ is not compact as an operator on BMOA.

By Theorem 12, for each $n \in \mathbb{N} \cup\{0\}, \varphi^{\prime}(0)^{n}$ is an eigenvalue of $C_{\varphi}$ as an operator acting on any of the spaces $\mathscr{B}_{H}^{\alpha}, \mathscr{A}_{H}^{-\alpha}, \mathscr{Z}_{H}, B_{H}^{p}$, and $\mathrm{BMOA}_{H}$, and the corresponding eigenfunctions are given by $\alpha \sigma^{n}+\bar{\beta} \bar{\sigma}^{n}$, where $\alpha$ and $\beta$ are arbitrary complex constants, not both zero.

5.1. Concluding Remarks. We wish to point out that since harmonicity is not preserved under multiplication unless the multiplier is constant, multiplication operators are of no interest for the operator theory of spaces of harmonic mappings.

Composition operators on spaces of harmonic mappings could be expanded to include those induced by antianalytic self-maps of $\mathbb{D}$. We did not pursue this study as we expect that results for this case can easily be obtained from the analytic symbol case using appropriate transformations.

\section{Data Availability}

The findings in this research do not make use of data.

\section{Conflicts of Interest}

The authors declare that they have no conflicts of interest.

\section{References}

[1] D. Girela, "Analytic functions of bounded mean oscillation, Complex function spaces (Mekrijärvi, 1999)," vol. 4, in Department of Mathematics Report Series, pp. 61-170, University of Joensuu, Joensuu, Finland, 2001.

[2] M. Aljuaid, "The operator theory on some spaces of harmonic mappings," Doctoral Dissertation, George Mason University, Fairfax, Virginia, 2019.

[3] K. Zhu, "Bloch type spaces of analytic functions," Rocky Mountain Journal of Mathematics, vol. 23, no. 3, pp. 11431177, 1993.

[4] M. Aljuaid and F. Colonna, "Characterizations of Bloch-type spaces of harmonic mappings," Journal of Function Spaces, vol. 2019, Article ID 5687343, 11 pages, 2019.

[5] M. Aljuaid and F. Colonna, On the Harmonic Zygmund Spaces, Preprint, Bulletin of the Australian Mathematical Society, Cambridge, UK.

[6] F. Colonna, "The Bloch constant of bounded harmonic mappings," Indiana University Mathematics Journal, vol. 38, no. 4, pp. 829-840, 1989.

[7] H. Hedenmalm, B. Korenblum, and K. Zhu, Theory of Bergman Spaces, Springer-Verlag, New York, NY, USA, 2000.

[8] J. Arazy and S. D. Fisher, "Some aspects of the minimal, Möbius-invariant space of analytic functions on the unit disc," in Interpolation Spaces and Allied Topics in Analysis (Lund). Lecture Notes in Math. 1070, Vol. 24-44, Springer, Berlin, Germany, 1984.

[9] J. Arazy, S. D. Fisher, and J. Peetre, "Möbius invariant function spaces," Journal für die reine und angewandte Mathematik, vol. 363, pp. 110-145, 1985.

[10] M. Tjani, "Compact composition operators on some Moebius invariant Banach spaces," Ph.D. thesis, Michigan State University, East Lansing, MI, USA, 1996.

[11] K. Madigan and A. Matheson, "Compact composition operators on the Bloch space," Transactions of the American Mathematical Society, vol. 347, no. 7, pp. 2679-2687, 1995.

[12] J. Xiao, "Composition operators associated with Bloch-type spaces," Complex Variables, Theory and Application: An International Journal, vol. 46, no. 2, pp. 109-121, 2001.

[13] S. Ye and Q. Hu, "Weighted composition operators on the Zygmund space," Abstract and Applied Analysis, vol. 2012, Article ID 462482, 18 pages, 2012.

[14] M. Tjani, "Compact composition operators on Besov spaces," Transactions of the American Mathematical Society, vol. 355, no. 11, pp. 4683-4698, 2003. 
[15] F. Colonna and S. Li, "Weighted composition operators from the minimal Möbius invariant space into the Bloch space," Mediterranean Journal of Mathematics, vol. 10, no. 1, pp. 395-409, 2013.

[16] J. Laitila, "Weighted composition operators on BMOA," Computational Methods and Function Theory, vol. 9, no. 1, pp. 27-46, 2009.

[17] W. Smith, "Compactness of composition operators on BMOA," Proceedings of the American Mathematical Society, vol. 127, no. 9, pp. 2715-2725, 1999.

[18] H. Wulan, D. Zheng, and K. Zhu, "Compact composition operators on BMOA and the Bloch space," Proceedings of the American Mathematical Society, vol. 137, no. 11, pp. 38613868, 2009.

[19] F. Colonna and M. Tjani, "Operator norms and essential norms of weighted composition operators between Banach spaces of analytic functions," Journal of Mathematical Analysis and Applications, vol. 434, no. 1, pp. 93-124, 2016.

[20] J. Bonet, M. Lindström, and E. Wolf, "Isometric weighted composition operators on weighted Banach spaces of type $H^{\infty}$," Proceedings of the American Mathematical Society, vol. 136, no. 12, pp. 4267-4273, 2008.

[21] F. Colonna and N. Hmidouch, "Weighted composition operators on iterated weighted-type Banach spaces of analytic functions," Complex Analysis and Operator Theory, vol. 13, no. 4, pp. 1989-2016, 2019.

[22] M. Shabazz and M. Tjani, "Isometries among composition operators on Besov type spaces," Complex Variables and Elliptic Equations, vol. 61, no. 6, pp. 761-771, 2016.

[23] N. Zorboska, "Isometric composition operators on the Blochtype spaces," Comptes Rendus Mathematiques de l'Académie des Sciences. La Société Royale du Canada, vol. 29, no. 3, pp. 91-96, 2007.

[24] R. F. Allen, K. C. Heller, and M. A. Pons, "Isometric composition operators on the analytic Besov spaces," Journal of Mathematical Analysis and Applications, vol. 414, no. 1, pp. 414-423, 2014.

[25] G. Bao and H. Wulan, "The minimal Möbius invariant space," Complex Variables and Elliptic Equations, vol. 59, no. 2, pp. 190-203, 2014.

[26] J. H. Shapiro, Composition operators and Classical Function Theory, Universitext: Tracts in Mathematics, Springer-Verlag, New York, NY, USA, 1993.

[27] J. Laitila, "Isometric composition operators on BMOA," Mathematische Nachrichten, vol. 283, no. 11, pp. 1646-1653, 2010.

[28] F. Colonna, "Characterisation of the isometric composition operators on the Bloch space," Bulletin of the Australian Mathematical Society, vol. 72, no. 2, pp. 283-290, 2005.

[29] J. M. Cohen and F. Colonna, "Preimages of one-point sets of Bloch and normal functions," Mediterranean Journal of Mathematics, vol. 3, no. 3-4, pp. 513-532, 2006.

[30] M. J. Martín and D. Vukotić, "Isometries of the Dirichlet space among the composition operators," Proceedings of the American Mathematical Society, vol. 134, no. 6, pp. 1701-1705, 2006.

[31] C. N. B. Hammond, "On the norm of a composition operator," Doctoral Dissertation, University of Virginia, Charlottesville, Virginia, 2003.

[32] B. Paudyal, "Eigenfunctions of composition operators on bloch-type spaces," Doctoral Dissertation, The University of Toledo, Toledo, OH, USA, 2014.

[33] P. S. Bourdon, J. A. Cima, and A. L. Matheson, "Compact composition operators on the BMOA," Transactions of the American Mathematical Society, vol. 351, no. 6, pp. 21832196, 1999. 